\title{
IDENTIFICAÇÃO DOS FATORES DE RISCO PARA O TRANSTORNO DO PROCESSAMENTO AUDITIVO (CENTRAL) EM PRÉ-ESCOLARES
}

\author{
Identification of risk factors for the (central) auditory processing \\ disorder in preschool children
}

\author{
Daniela Musskopf da Luz ${ }^{(1)}$, Maria Inês Dornelles da Costa-Ferreira ${ }^{(2)}$
}

\begin{abstract}
RESUMO
Objetivos: identificar os fatores de risco para o Transtorno do Processamento Auditivo (Central) TPA(C) em 79 pré-escolares. Método: aplicou-se um questionário para 79 pais, de crianças que cursavam a pré-escola ou o primeiro ano do ensino fundamental, das redes municipal, estadual e particular de Porto Alegre. O questionário continha questões referentes ao desenvolvimento infantil. Resultados: a pesquisa mostra como principais resultados a ocorrência do uso de chupeta $(73,4 \%)$; do tempo de aleitamento materno (36\%); da realização dos exames audiológicos (17,8\%); do tempo em frente a televisão (59\%); e da quantidade de repetições de instruções (54\%); Em relação aos testes de correlação o estudo mostrou a utilização da chupeta $(p=0,006)$ e maior ocorrência de otites $(p=0,007)$ por meninas. As crianças mais velhas realizavam leitura espontânea $(p=0,007)$ e recontavam histórias ordenadamente $(p=0,035)$. Conclusão: Identificou-se as variáveis: maior número de irmãos $(p=0,005)$, maior solicitação de repetições $(p<0,001)$ e dificuldade de compreensão em ambientes competitivos $(p=0,002)$ significativos como fatores de risco para o Transtorno do Processamento Auditivo (Central).
\end{abstract}

DESCRITORES: Perda Auditiva Central; Fatores de Risco; Desenvolvimento Infantil; Cognição; Pré-Escolar

\section{INTRODUÇÃO}

As experiências auditivas têm um papel importante na capacidade de comunicação e, por sua vez, na compreensão e a na socialização. Nesse contexto, o processamento auditivo central $\mathrm{PA}(\mathrm{C})$ é a eficiência e a efetividade com que o sistema nervoso central utiliza a informação auditiva'. Diante de alguma intercorrência no processo maturacional, dificuldades podem ser observadas em algumas habilidades auditivas principalmente quando a

(1) Fonoaudióloga pelo Centro Universitário Metodista do Instituto Porto Alegre, Porto Alegre, RS, Brasil.

(2) Fonoaudióloga; Doutora pela Pontifícia Universidade Católica do Rio Grande do Sul - PUCRS, Porto Alegre, RS, Brasil; Professora do curso de Fonoaudiologia do Centro Universitário Metodista do Instituto Porto Alegre, Porto Alegre,RS; Professora da Faculdade Nossa Senhora de Fátima, Caxias do Sul,RS, Brasil.

Conflito de interesses: inexistente criança se encontra em idade escolar configurando um Transtorno do Processamento Auditivo (Central) [TPA(C)]. Dessa forma, o convívio com outras crianças ou adultos proporciona a observação do impacto nas situações comunicativas, na socialização, bem como, em outros aspectos necessários à aprendizagem como a cognição.

Quando há prejuízo das experiências sonoras, como consequência das deficiências auditivas adquiridas, principalmente causadas pelas otites de repetição, geralmente, observa-se a influência na aquisição da linguagem oral e na aprendizagem, pois a presença desse quadro não inviabiliza o desenvolvimento da linguagem, mas a compromete, alterando a discriminação da fala, principalmente em ambientes ruidosos, o que pode comprometer também, as habilidades de consciência fonológica e por consequência, o desempenho escolar ${ }^{2,3}$. Outro fator que pode ser agravante da perda auditiva condutiva é a ingestão de líquidos na posição 
deitada, principalmente em bebês além de configurar um hábito oral deletério ${ }^{4}$ poderá contribuir para um quadro de respiração oral e este, a longo prazo, encontrar-se associado aos transtornos do sono e/ou da atenção e até das alterações neurocognitivas gerais ${ }^{5,6}$. Também é possível suspeitar de alterações nas habilidades auditivas que podem ser confirmadas através dos testes comportamentais e/ou das medidas eletrofisiológicas levando ao diagnóstico do TPA $(C)$. Além disso, hábitos como: limpar as orelhas com hastes flexíveis ou outros objetos, ouvir música em intensidade elevada e usar fones de ouvido, também são prejudiciais à audição ${ }^{7}$.

A prematuridade principalmente se acompanhada de baixo peso ao nascer também configura um fator de risco para os transtornos auditivos cujos indicadores de risco são: internação do bebê em UTI por período igual ou superior a 48hs, síndromes, histórico familiar, anomalias crânio faciais e infecções congênitas ${ }^{8}$. Tais indicadores são considerados fatores etiológicos de perda auditiva periférica ou TPA $(\mathrm{C})^{1}$.

As substituições na fala envolvendo os sons $/ \mathrm{r} /$ e $/ \mathrm{I} /$, as inversões de letras, as dificuldades na compreensão leitora e as dificuldades de atenção em ambientes ruidosos são exemplos das principais manifestações comportamentais do TPA(C) ${ }^{9}$ que pode se encontrar relacionado a problemas fonológicos, de aprendizado e de linguagem decorrentes da dificuldade no processamento de estímulos acústicos. Assim, algumas crianças necessitam de mais tempo para processar os estímulos sonoros ${ }^{10 .}$

As funções cognitivas como a atenção, a percepção, a memória, a linguagem e as funções executivas também são essenciais ao desenvolvimento e à aprendizagem da criança. Nesse âmbito, as dificuldades de atenção influenciam no registro das informações na memória de trabalho implicando em maior tempo para a realização das tarefas escolares ${ }^{11}$.

O diagnóstico precoce dos transtornos de aprendizagem em pré-escolares, pode beneficiar a criança, auxiliando-a a superar as dificuldades nas atividades de leitura e escrita através de uma abordagem terapêutica adequada ao caso ${ }^{12}$. Além disso, destacam-se atividades facilitadoras que podem ser utilizadas em casa, na escola ou em outros contextos. A contação de histórias, por exemplo, é uma atividade que auxilia no desenvolvimento cognitivo geral, na melhora da compreensão e na ampliação do vocabulário. Conhecer como ocorre a narrativa oral das histórias é essencial ao processo de alfabetização e desenvolvimento da escrita ${ }^{13}$. O brincar e a música também são formas lúdicas que a criança encontra para simbolizar e, consequentemente, crescer e se desenvolver.

Outro aspecto que se encontra relacionado à leitura e à escrita das crianças é a nomeação rápida em que, ao processar a informação rapidamente, nomeando e convertendo o grafema ao seu som, a criança demonstra maior habilidade nas tarefas de leitura ${ }^{14}$.

De acordo com os aspectos apresentados, o objetivo do presente estudo é identificar os fatores de risco para o Transtorno do Processamento Auditivo (Central) TPA(C) em 79 pré-escolares.

\section{MÉTODO}

Trata-se de um estudo transversal, de grupo e contemporâneo. O instrumento de estudo é um questionário que busca reunir a maioria dos aspectos apresentados na literatura e/ou observados na clínica fonoaudiológica que possam configurar fatores de risco para o TPA $(C)$ cujas questões envolvem os aspectos referentes à gestação, hábitos orais, sinais relacionados às queixas auditivas, desenvolvimento linguístico, socialização e aspectos cognitivos. O desfecho esperado é o conhecimento do perfil destes aspectos nas crianças em idade pré-escolar e sua relação com os fatores de risco para o TPA(C).

A população da presente pesquisa foi constituída pelos responsáveis dos estudantes da educação infantil e do primeiro ano do ensino fundamental de escolas particulares, estaduais e municipais do município de Porto Alegre. A inclusão das turmas de primeiro ano deve-se ao fato das mesmas não possuírem experiência prévia na pré-escola. A realização dessa pesquisa foi vinculada à assinatura do Termo de Conhecimento Institucional (TCl), por todas as escolas participantes da pesquisa. Após, todos os responsáveis pelos alunos matriculados na educação infantil e no primeiro ano do ensino fundamental foram convidados a participar do estudo, exceto aqueles que não assinaram o Termo de Consentimento Livre e Esclarecido (TCLE), configurando, assim, os critérios de exclusão. Todos os participantes da pesquisa foram devidamente esclarecidos sobre seus propósitos.

Como todos os indivíduos desta população foram incluídos, o cálculo do tamanho da amostra não foi realizado.

A coleta dos dados deu-se nos meses de junho a setembro de 2009 , em que foi aplicado um questionário a todos os responsáveis pelas crianças das escolas pesquisadas que aceitaram participar da pesquisa, totalizando 79 entrevistas. O questionário, Figura 1, foi elaborado pelas autoras, baseado nos sinais relacionados às queixas auditivas (otites de 


\section{Aspectos Audiológicos e Cognitivos em Pré-Escolares}

Nome:

Sexo: M ( ) F( ) Idade:

Escola:

Série:

Responsável:

Aspectos da gestação, nascimento e desenvolvimento.

1. Houve algum problema durante a gestação? ( ) S ( ) N

2. Caso afirmativo, descrever quais problemas e causas.

3. Foi amamentado no peito? () S () N Qto tempo () 6 meses () 6 meses ()6 a 12 meses () mais de 1 ano

4. Faz uso de mamadeira? ( ) $\mathrm{S}$ ( ) $\mathrm{N}$ bico? ( ) $\mathrm{S}$ ( ) $\mathrm{N}$

5. Costuma ingerir líquido deitado? () $\mathrm{S}$ ( ) $\mathrm{N}$

6. Tem algum problema de saúde? () S ( ) N Qual?

7. Toma alguma medicação? ( ) S ( ) N

8. Há algum parente com problemas auditivos? ( ) S ( ) N

9. Costuma ter otites? () S ( ) N Qual frequência?

10. Começou a falar as primeiras palavras com que idade? ( ) 1 ano ( ) 1-2 anos ( ) 2-3 anos ( ) mais de 3 anos

11. Conhece cores? () S ( ) N Nomeia as cores? ( ) S ( ) N

12. Você acha que seu filho escuta bem? ( ) S ( ) N

13. Seu filho já fez algum exame audiológico? ( ) S ( ) N Qual?

Aspectos familiares e rotina.

14. Seu filho tem irmãos? () S ( ) N ( ) mais velhos ( ) mais novos ( ) os dois

15. Convive com eles? ( ) S ( ) N

16. Seu filho costuma conversar mais com crianças ou adultos? ( ) C ( ) A

17. Como são suas brincadeiras? ( ) miniaturas ( ) sucatas () brinquedos barulhentos ( ) montar

18. Brinca com outras crianças? ( ) rua () escola ( ) casa

19. Gosta de ouvir histórias? () S ( ) N

20. Com que freqüência alguém conta história $\mathrm{p} /$ cça? ( ) todos os dias ( ) 1x/sem ( )+ 1x/sem ( ) de vez em quando

21. Presta atenção na história? ( ) S ( ) N

22. Consegue ler sozinha a história? ( ) S ( ) N

23. Gosta de manusear livros? ( ) S ( ) N

24. Assiste televisão? ( ) S ( ) N Tempo: ( ) $1 \mathrm{~h} \mathrm{(} \mathrm{)} \mathrm{1-} 2$ hs ( ) + de 2 hs

25. Gosta de escutar música? () S ( ) N Tipo:

26. Consegue recontar as histórias? ( ) S ( ) N ( ) na ordem () fora de ordem ( ) não consegue

27. Quando assiste tv ou escuta música o volume é alto? ( ) S ( ) N

28. Sente incomodo com som/ ruído forte? ( ) S ( ) N

29. Costuma ser distraído a ponto de não atender ao chamado? ( ) S ( ) N em que situações?

30. Demora em responder perguntas?

31. Costuma pedir repetição de ordens/ perguntas? () S ( ) N ( ) sempre () as vezes () nunca

32. Entende o que é falado mesmo quando há mais pessoas falando ao mesmo tempo? ( ) S ( ) N

33. Costuma usar fones de ouvido? ( ) S ( ) N

34. Fala lentamente ou rápido demais? $L() R()$

35. Sabe diferenciar: ( ) em cima ( ) em baixo ( ) do lado ( ) dentro ( ) fora ( ) n sabe

36. Tem conceitos como: ( ) semana () dia () mês () n sabe

37. Consegue memorizar: ( ) nomes ( ) lugares ( ) itinerários ( ) n consegue

38. Durante o sono: ( ) ronca ( ) baba () ronca e baba

39. Vai a escola desde que idade?

Questionário baseado nos livros dos autores: AZEVEDO et al.1995; AZEVEDO, 1996; PEREIRA, 1996.

Figura 1 - Aspectos audiológicos e cognitivos em escolares 
repetição, volume alto, entender ordens, pedir repetição de ordens) descritos por alguns autores como fatores de risco para o TPA(C) ${ }^{9,15,16}$. Além disso, outras questões foram acrescentadas referentes à gestação e à amamentação (intercorrências e tempo), à cognição (memória, atenção, percepção, linguagem) ao desenvolvimento linguístico à socialização. As questões elaboradas também foram relacionadas às variáveis gênero, idade, nível de educação infantil e rede de ensino.

As pesquisadoras contaram com o auxílio de quatro colegas, que, depois do devido treinamento, de observação e esclarecimentos sobre as perguntas, se encontravam aptas para a coleta dos dados. Todas as coletas foram realizadas de modo presencial com os responsáveis no horário de entrada ou saída das crianças nas escolas.

A presente pesquisa foi aprovada pelo Comitê de Ética em Pesquisa do Centro Universitário Metodista IPA, sob protocolo 123/2008 e faz parte de um projeto mais amplo.

Para descrever o perfil da amostra segundo as variáveis em estudo, foram realizadas as tabelas de freqüência das variáveis categóricas com valores de freqüência absoluta (n) e percentual (\%) e estatísticas descritivas das variáveis contínuas com valores de média, desvio padrão, valores mínimo e máximo, mediana e quartis. Para a comparação das variáveis categóricas entre os grupos, foram utilizados os testes Qui-Quadrado ou exato de Fisher (para valores esperados menores que 5); para comparar as variáveis numéricas entre dois grupos foi utilizado o teste de Mann-Whitney; para comparar as variáveis numéricas entre trêsgrupos ou mais foi utilizado o teste de Kruskal-Wallis, devido à ausência de distribuição normal; para analisar a relação entre as variáveis numéricas foi utilizado o coeficiente de correlação de Spearman, devido à ausência de distribuição normal das variá- veis. O nível de significância adotado para os testes estatísticos foi de $5 \%$, ou seja, $\mathrm{P}$ por $\mathrm{p}<0,05$.

\section{RESULTADOS}

O presente estudo constitui-se de 79 entrevistas fornecidas pelos pais e/ou responsáveis de crianças que frequentavam a pré-escola ou o primeiro ano do ensino fundamental. Dessas cianças, $39(49,4 \%)$ eram meninos e 40 (50,6\%), meninas.

Em relação à idade, $14(17,7 \%)$ tinham 4 anos de idade; 31 (39,2\%), 5 anos; 19 (24,1\%), 6 anos; 11 (13,9\%), 7 anos; 3 (3,8\%), 8 anos e $1(1,3 \%)$ tinha 9 anos de idade.

Considerando-se a série, 22 crianças $(27,9 \%)$ cursavam o Jardim A; 31 (39,2\%), o Jardim B e 26 $(32,9 \%)$ cursavam o primeiro ano do ensino fundamental. Da mesma forma, 21 crianças $(26,6 \%)$ frequentavam a rede particular de ensino; 32 $(40,5 \%)$, a rede municipal e $26(32,9 \%)$ frequentavam a rede estadual de ensino.

A Tabela 1 mostra as variáveis referentes aos aspectos de saúde, alimentação e hábitos orais deletérios em relação a sua freqüência. Além dos resultados apresentados na Tabela 1, os familiares identificaram como causa da intercorrência gestacional, ao considerar os nove casos, a diabetes apresentada por três $(33,3 \%)$ mães; a prematuridade em três $(33,3 \%)$ crianças; a gravidez indesejada em uma $(11,1 \%)$ mãe; a utilização de drogas em uma $(11,1 \%)$ mãe e a ocorrência de infecções em uma $(11,1 \%)$ criança.

Dezoito dos responsáveis entrevistados referiram problemas de saúde em seu filho, encontrando-se: cinco $(27,8 \%)$, com asma; seis $(33,3 \%)$, com bronquite; quatro $(22,2 \%)$, com sinusite e três $(16,7 \%)$, com rinite.

Em relação ao tempo de aleitamento materno, $24(32 \%)$, mães amamentaram por um tempo inferior a seis meses; seis (8\%) amamentaram por

Tabela 1 - Frequência das variáveis de saúde, alimentação e hábitos orais deletérios ( $n=79)$

\begin{tabular}{lcccc}
\hline & \multicolumn{2}{c}{ PRESENÇA } & \multicolumn{2}{c}{ AUSÊNCIA } \\
\cline { 2 - 5 } & $\mathbf{n}$ & $\mathbf{( \% )}$ & $\mathbf{n}$ & $\mathbf{( \% )}$ \\
\hline Intercorrência gestacional & 9 & 11,4 & 70 & 88,6 \\
Aleitamento materno & 75 & 94,9 & 4 & 5,1 \\
Aleitamento artificial & 41 & 51,9 & 38 & 48,1 \\
Utilização de chupeta & 21 & 26,6 & 58 & 73,4 \\
Ingestão de líquido deitado & 35 & 44,3 & 44 & 55,7 \\
Problemas de saúde & 18 & 22,8 & 61 & 77,2 \\
Utilização de medicação & 4 & 5,1 & 75 & 94,9 \\
\hline
\end{tabular}

$\mathrm{n}=$ frequência absoluta; $\%=$ percentual 
seis meses; 18 (24\%) amamentaram de seis a doze meses e 27 (36\%) amamentaram por um tempo superior a doze meses.

Quando questionados em relação ao sono, 41 $(51,9 \%)$, responsáveis referiram que o filho apresentou sono normal; $14(17,7 \%)$ apontaram que o filho roncava; oito $(10,1 \%)$ referiram que o filho babava e $16(20,3 \%)$ mencionaram que o filho roncava e babava.

A Tabela 2 apresenta as variáveis referentes às queixas audiológicas em relação à presença ou ausência das mesmas.

Das crianças que realizaram avaliação audiológica, $13(92,9 \%)$ realizaram audiometria, e apenas uma $(7,1 \%)$ realizou o teste da orelhinha.

A frequência dos aspectos familiares e sociais é apresentada na Tabela 3.

Em relação aos aspectos familiares, ao considerar as 52 crianças que possuem irmãos, 34 $(65,4 \%)$ tinham irmãos mais velhos; nove $(17,3 \%)$ tinham irmãos mais novos e nove $(17,3 \%)$ tinham irmãos mais velhos e mais novos.

Quando questionados se os filhos conversavam mais com crianças ou adultos, $10(12,7 \%)$ pais referiram que os filhos conversavam mais com crianças;
32 (40,5\%), referiram que os filhos conversam mais com adultos e 37 (46,8\%), com ambos.

Das 78 crianças que assistem à televisão, 15 $(19,2 \%)$ assistia até uma hora; $17(21,8 \%)$ de uma a duas horas e $46(59,0 \%)$ assistem por tempo superior a duas horas por dia.

Em relação ao brincar, 22 (27,9\%) crianças brincavam somente na escola; 12 (15,2\%) brincavam em casa, e $45(57,0 \%)$ crianças brincavam em casa e na escola. Em relação aos brinquedos preferidos, constatou-se que: 11 (13,9\%) crianças preferiam brincar com miniaturas; uma $(1,3 \%)$ com sucatas; três $(3,8 \%)$ com brinquedos sonoros; seis $(7,6 \%)$ com brinquedos de encaixe e $58(73,4 \%)$ com mais de uma opção de brinquedos.

A seguir, serão abordados os aspectos lingüísticos. O primeiro é a época do surgimento das primeiras palavras, sendo: $44(55,7 \%)$ crianças falaram com menos de um ano de idade; $24(30,4 \%)$ falaram entre um e dois anos de idade; oito $(10,1 \%)$ falaram entre dois e três anos de idade e três $(3,8 \%)$ com mais de três anos de idade.

A Tabela 4 apresenta outros aspectos relacionados ao desenvolvimento da linguagem, considerados relevantes.

Tabela 2 - Presença e ausência de queixas audiológicas ( $n=79)$

\begin{tabular}{lcccc}
\hline & \multicolumn{2}{c}{ PRESENÇA } & \multicolumn{2}{c}{ AUSÊNCIA } \\
\cline { 2 - 5 } & $\mathbf{n}$ & $\mathbf{( \% )}$ & $\mathbf{n}$ & (\%) \\
\hline Parente c/problema auditivo & 21 & 26,6 & 58 & 73,4 \\
Acuidade auditiva da criança & 75 & 94,9 & 4 & 5,1 \\
Realizou exame audiológico & 14 & 17,7 & 65 & 82,3 \\
Otites & 17 & 21,5 & 62 & 78,5 \\
Preferência por volume alto & 36 & 45,6 & 43 & 54,4 \\
Incômodo a sons fortes & 26 & 32,9 & 53 & 67,1 \\
Utilização de fones de ouvido & 9 & 11,4 & 70 & 88,6 \\
Compreensão em ambientes & 68 & 86,1 & 11 & 13,9 \\
competitivos & & & & \\
\hline
\end{tabular}

$\mathrm{n}=$ frequência absoluta; $\%=$ percentual

Tabela 3 - Frequência dos aspectos familiares e sociais $(n=79)$

\begin{tabular}{lcccc}
\hline & \multicolumn{2}{c}{ PRESENÇA } & \multicolumn{2}{c}{ AUSÊNCIA } \\
\cline { 2 - 5 } & $\mathbf{n}$ & $\mathbf{( \% )}$ & $\mathbf{n}$ & (\%) \\
\hline Irmãos & 52 & 65,8 & 27 & 34,2 \\
Convive com irmãos & 43 & 82,7 & 9 & 17,3 \\
Assiste televisão & 78 & 98,7 & 1 & 1,3 \\
\hline
\end{tabular}

$\mathrm{n}=$ frequência absoluta; $\%=$ percentual 
Tabela 4 - Frequência dos aspectos lingüísticos referidos no questionário ( $n=79)$

\begin{tabular}{lcccc}
\hline & \multicolumn{2}{c}{ PRESENÇA } & \multicolumn{2}{c}{ AUSÊNCIA } \\
\cline { 2 - 5 } & $\mathbf{n}$ & $\mathbf{( \% )}$ & $\mathbf{n}$ & $\mathbf{( \% )}$ \\
\hline Gosta de ouvir histórias & 76 & 96,2 & 3 & 3,8 \\
Atenção na história & 72 & 91,1 & 7 & 8,9 \\
Leitura espontânea de história & 11 & 13,9 & 68 & 86,1 \\
Manuseio de livros & 74 & 93,7 & 5 & 6,3 \\
Reconto de histórias & 75 & 94,9 & 4 & 5,1 \\
Reconto de história ordenadamente & 62 & 82,7 & 13 & 17,3 \\
\hline
\end{tabular}

$\mathrm{n}=$ frequência absoluta; \%= percentual

A frequência das histórias contadas para as crianças diariamente foi apontada por $20(25,3 \%)$ pais. Já $17(21,5 \%)$ contavam histórias aos filhos semanalmente; 11 (13,9\%), contavam histórias mais de uma vez por semana, e 31 (39,3\%), referiram contar histórias esporadicamente.

Por fim, a Tabela 5 evidencia aspectos cognitivos relevantes ao desenvolvimento infantil.

Tabela 5 - Frequência dos aspectos cognitivos referidos no questionário $(n=79)$

\begin{tabular}{lcccc}
\hline & \multicolumn{2}{c}{ PRESENÇA } & \multicolumn{2}{c}{ AUSÊNCIA } \\
\cline { 2 - 5 } & $\mathbf{n}$ & $\mathbf{( \% )}$ & $\mathbf{n}$ & $\mathbf{( \% )}$ \\
\hline Conhecimento das cores & 75 & 94,9 & 4 & 5,1 \\
Nomeação das cores & 72 & 91,1 & 7 & 8,9 \\
Identificação das noções espaciais & 73 & 92,4 & 6 & 7,6 \\
Distração ao ser chamado & 38 & 48,1 & 41 & 51,9 \\
Demora p/ responder perguntas & 24 & 30,4 & 55 & 69,6 \\
Solicita repetição de instruções & 41 & 51,9 & 38 & 48,1 \\
Memória para nomes & 78 & 98,7 & 1 & 1,3 \\
Memória para locais & 67 & 84,8 & 12 & 15,2 \\
Memória para itinerários & 61 & 77,2 & 18 & 22,8 \\
\hline
\end{tabular}

$\mathrm{n}=$ frequência absoluta; \%= percentual

Em relação à frequência da repetição de instruções, apresentada em 41 crianças, 19 (46,3\%) sempre solicitavam repetição e $22(53,7 \%)$ solicitavam algumas vezes. Ao se questionarem os pais sobre a situação em que o filho apresentava distração, $12(31,6 \%)$ referiram a televisão; oito $(21,1 \%)$ as brincadeiras, $17(44,7 \%)$ apontaram que o filho sempre se mostra distraído, e apenas um $(2,6 \%)$ apontou que o filho distraia-se escutando música.

A seguir, serão apresentadas as análises comparativas das variáveis destacadas entre os gêneros, idade, níveis de educação infantil e análise comparativa do número de fatores de risco.

A primeira análise realizada comparou as variáveis estudadas entre os gêneros. A variável idade mostrou-se estatisticamente significativa ao identificar a maior frequência de meninas com seis anos ou mais $(p=0,047)$ sendo esta, uma característica da amostra. Nessa comparação, a Figura 2 mostra relação estatisticamente significativa para a utilização de chupeta $(p=0,006)$ e frequência de otites $(p=0,016)$ em meninas.

Em relação à análise comparativa entre as faixas etárias, identificou-se relação estatisticamente significativa entre esta variável e a série $(p=<0,001)$. Das 14 crianças com 4 anos de idade, 13 (92,7\%) frequentavam o Jardim A. Da mesma forma, das 50 crianças de cinco e seis anos de idade, 30 (60\%) frequentavam o Jardim B e as $15(100 \%)$ crianças com sete anos ou mais frequentavam o primeiro ano do ensino fundamental. Das 14 crianças com quatro anos de idade, nove $(64,3 \%)$ frequentavam a escola municipal, da mesma forma que 23 (46\%) crianças de cinco e seis anos de idade num total de 50 crianças. Já a escola estadual foi frequentada por $15(100 \%)$ crianças. Tal relação foi estatisticamente significativa $(p=<0,001)$. 

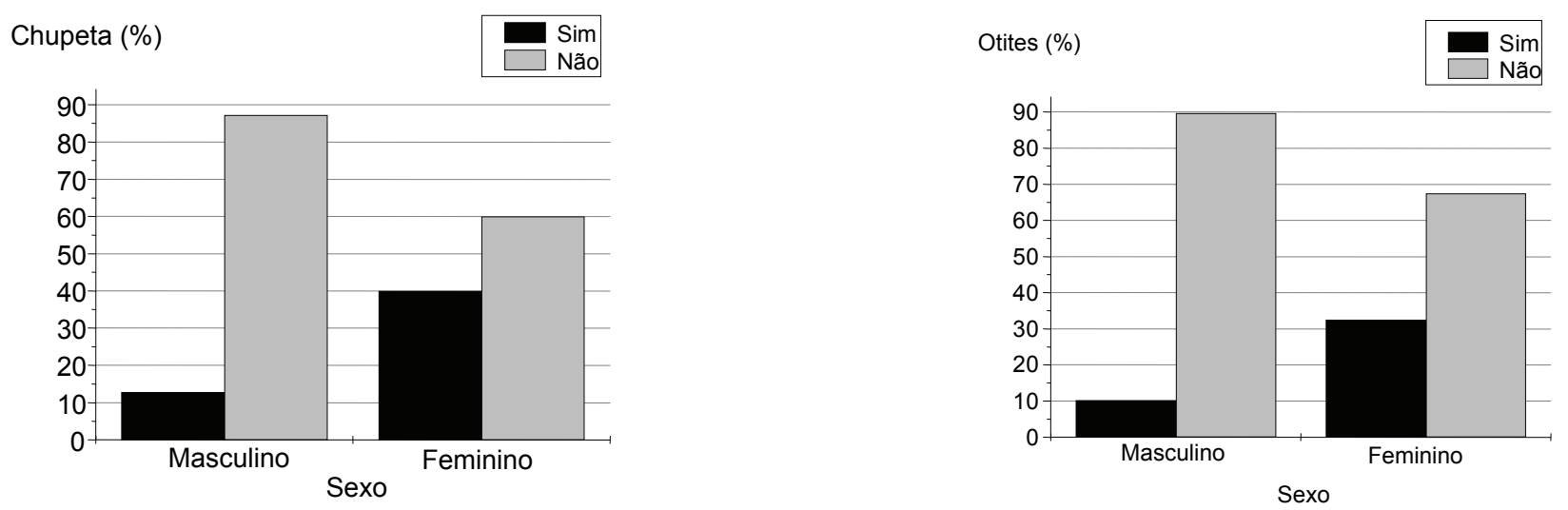

Figura 2 - Utilização de chupeta e frequência de otites entre os gêneros

Outro aspecto abordado é a presença de irmãos, que foi estatisticamente significativa em crianças mais velhas $(p=0,046)$. Estas, preferem desenvolver a conversação com adultos e crianças $(p=<0,001)$, optando também por brincadeira na escola e no lar $(p=0,045)$.

A Figura 3 mostra a distribuição das crianças em relação à leitura espontânea de histórias, sendo estatisticamente significativa $(p=0,007)$ para as crianças mais velhas.

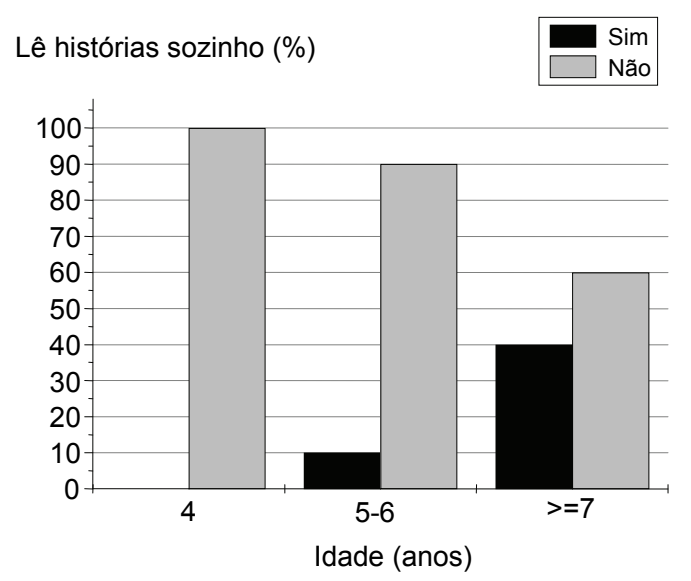

Figura 3 - Distribuição das crianças em relação à leitura espontânea considerando-se a idade

As crianças mais velhas recontam histórias ordenadamente se comparadas às crianças mais jovens $(p=0,035)$.

Ao se realizar a análise comparativa entre os níveis de educação, obteve-se maior frequência da realização de avaliação audiológica nas crianças matriculadas no Jardim A $(p=0,005)$. Estas também assistem até uma hora de televisão por dia $(p=0,021)$.
Já as crianças que frequentam o Jardim B e o primeiro ano do ensino fundamental recontam histórias ordenadamente $(p=<0,001)$, bem como, realizam sua leitura espontaneamente $(p=<0,001)$ como mostra a Figura 4.

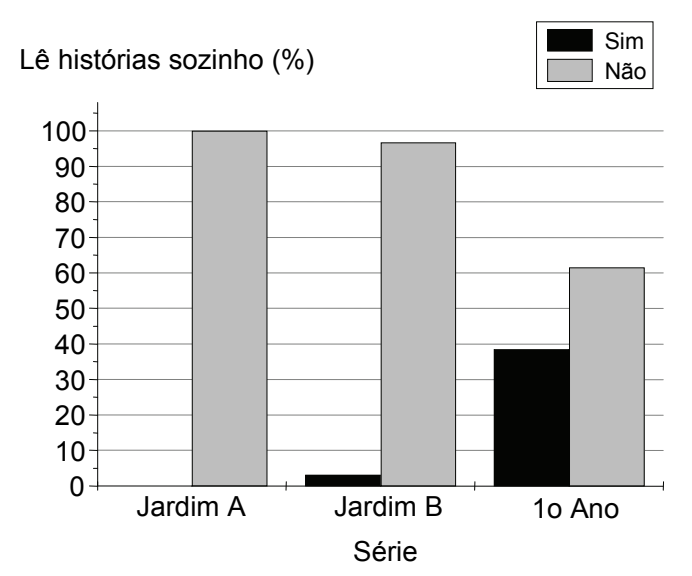

Figura 4 - Distribuição das crianças ao realizar a leitura espontânea considerando-se os níveis de educação

Posteriormente, identificaram-se as variáveis: intercorrência gestacional, ingestão de líquido na posição deitada, problemas de saúde, atraso na aquisição de linguagem, não conhecer e não nomear cores, não ter boa acuidade auditiva, não gostar de ouvir histórias, não prestar atenção na história, não recontar histórias, não gostar de escutar música ou preferir escutá-la em intensidade elevada, não atender ao chamado pelo nome, apresentar intercorrência no sono e não conseguir memorizar nomes, lugares ou itinerários, como fatores de risco para o TPA(C). Tais variáveis foram quantificadas a fim de permitir a comparação do 

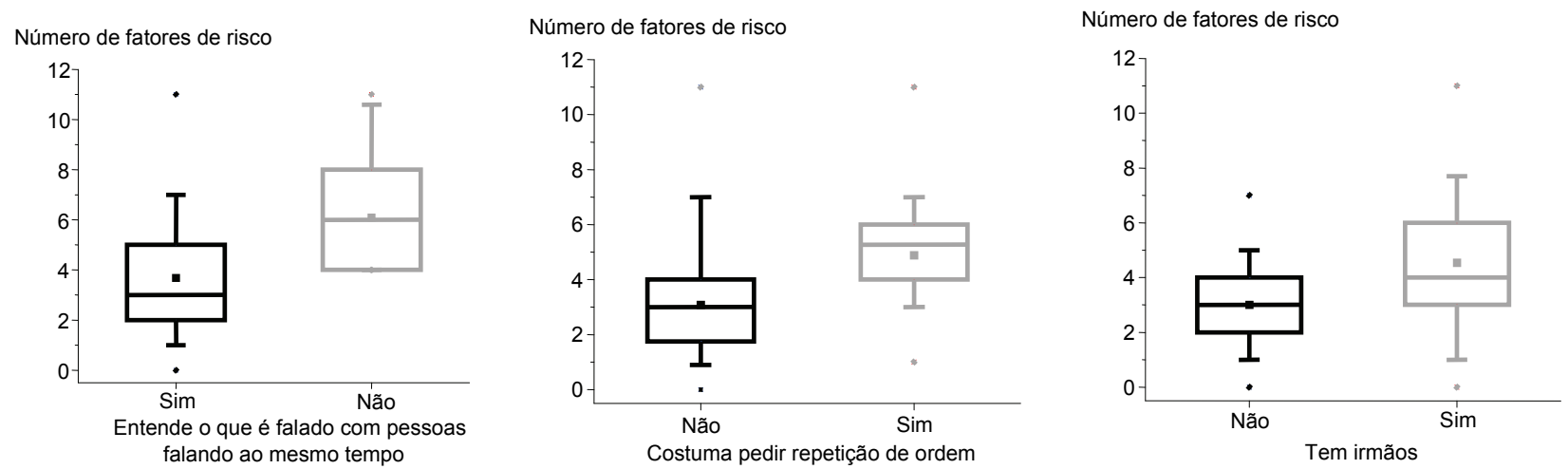

Figura 5 - Variáveis estatisticamente significantes em relação ao número de fatores de risco para o TPA(C)

número de fatores de risco com as demais variáveis. As variáveis presença de irmãos $(p=0,005)$, solicitação de repetição da instrução $(p=<0,001)$ e dificuldade de compreensão em ambientes competitivos $(p=0,002)$ foram estatisticamente significantes, sendo associadas ao maior número de fatores de risco, como mostra a Figura 5.

\section{DISCUSSÃO}

De acordo com o objetivo geral do estudo que é identificar fatores de risco para o TPA(C) em préescolares, optou-se por apresentar, nos resultados, as tabelas de frequência e análise descritiva das variáveis utilizadas no questionário.

Os resultados da pesquisa mostram que houve poucas intercorrências gestacionais, possibilitando verificar a eficiência do acompanhamento pré-natal, que tem por finalidade garantir a saúde da mãe e do feto durante a gestação e parto, identificando problemas que poderão gerar sequelas maternas ou infantis que aumentarão o risco de complicações ${ }^{17}$. No entanto, um estudo realizado com gestantes brasileiras mostra que as condições ofertadas de assistência durante a gestação e parto não são favoráveis, elevando a taxa de mortalidade infantil, principalmente neonatal ${ }^{18}$.

Em relação à amamentação, apesar dos resultados mostrarem tempo variável de aleitamento materno, prevaleceu a amamentação ao aleitamento artificial por mais de doze meses ${ }^{19}$. Esse fato nos remete à importância dos profissionais da saúde, como os fonoaudiólogos, que auxiliam as mães quanto à posição e pega correta para que não ocorram problemas nos seios, dificuldade referida pelas mães como causadoras do desmame precoce ${ }^{20}$. A amamentação exclusiva até os seis meses de idade também reduz o uso da chupeta ${ }^{21}$. A utilização da chupeta e da mamadeira constitui hábito oral deletério que pode causar deformidades anatômicas, favorecendo a respiração oral, além de ocasionar, com maior freqüência, a ocorrência de otites e infecções respiratórias. A otite constituem-se numa patologia que ocorre no componente condutivo, que, se for persistentes na infância pode ser prejudicial ao $\mathrm{PA}(\mathrm{C})$ mesmo após o tratamento, pois em época de aquisição da linguagem, a criança pode apresentar dificuldades na aquisição dos contrastes fonêmicos da língua configurando um TPA(C).

Quanto ao sono, a pesquisa mostra que metade das crianças têm sono normal, e a outra metade apresenta intercorrências. Tais crianças podem respirar pela boca à noite, prejudicando o sono, o que por sua vez pode influenciar no desempenho do $\mathrm{PA}(\mathrm{C}){ }^{5}$. Outro estudo mostra que os distúrbios respiratórios do sono acometem cerca de $12 \%$ das crianças, causando a respiração oral, e que estes fatores podem estar associados a rinites, asma e bronquite. Tais patologias foram referidas por 18 pais e não apresentam relação exclusivamente com o clima, pois estas dificuldades são apresentadas por crianças de todo Brasil ${ }^{22}$ sendo mais relacionadas às patologias da orelha média.

Outro dado a ser considerado é o baixo número de crianças que realizaram testes audiológicos, sendo um indicativo de que campanhas mais abrangentes devem ser realizadas para esclarecer sobre os riscos de alterações auditivas. O teste da orelhinha é de extrema importância para a detecção precoce de problemas auditivos. O ideal é que este teste seja realizado nas primeiras 48 horas de vida do bebê; destaca-se a necessidade de um trabalho interdisciplinar envolvendo outros profissionais da saúde, bem como medidas de saúde pública, como campanhas alertando aos pais sobre os riscos do bebê não apresentar boa acuidade auditiva ${ }^{2}$. O mesmo ocorre com a avaliação audiológica, pouco referida pelos pais, havendo necessidade de 
programas de prevenção, diagnóstico e intervenção da deficiência auditiva.

Campanhas dessa natureza poderiam integrar informações sobre hábitos que mais tarde também podem prejudicar a audição, como escutar música com volume muito alto, o que pode lesar o sistema auditivo ${ }^{7}$. Na pesquisa, a maioria das crianças não referiu este hábito, nem apresentou incômodo a sons fortes.

Em relação à compreensão em ambientes ruidosos, a maioria dos pais referiu que as crianças não apresentavam problemas. Se houvesse comprometimento do $\mathrm{PA}(\mathrm{C})$ a maioria das crianças apresentaria dificuldade em entender a conversa nestes ambientes ${ }^{9}$.

O hábito de assistir televisão por mais de duas horas diárias foi referido pela maioria dos responsáveis, o que vem ao encontro de outro estudo, ${ }^{23}$ que aponta que tal hábito está começando cada vez mais cedo nas crianças. Este resultado, juntamente com a referência de que as crianças costumam brincar mais na escola e em casa, aponta para a reflexão sobre outros aspectos atualmente identificados na sociedade, como a existência de programas de televisão mais voltados ao público infantil e a necessidade da manutenção das atividades profissionais da família, o que exige que as crianças permaneçam mais tempo na escola ou com cuidadores em casa.

As brincadeiras em casa costumam ser mais entre irmãos. Conforme os resultados apresentados na pesquisa, um grande número de crianças tem irmãos e convive eles. Um estudo revela que, quanto maior o número de filhos, maior o fator de risco para prontidão escolar ${ }^{24}$. Evidenciou-se na pesquisa, que essas crianças costumam conversar tanto com adultos quanto com outras crianças e suas brincadeiras são na escola e no lar.

Outro resultado significativo evidenciado na pesquisa é que a maioria das crianças começou a falar na época certa ${ }^{25}$ e gosta de escutar histórias. As histórias aumentam o vocabulário, auxiliando a desenvolver a linguagem e a atenção, bastante referida pelos pais quando questionados se os filhos prestavam atenção nas histórias. A atenção é essencial para a aprendizagem.

Embora não seja um resultado significativo, a pesquisa mostra que 41 crianças costumam solicitar repetição de instruções, talvez devido à sua pouca idade, pois as crianças mais velhas apresentam maior entendimento dos elementos lingüísticos que não estão diretamente ligados ao contexto imediato ${ }^{25}$. Do mesmo modo, os pais referiram que os filhos costumam se "distrair" olhando televisão ou brincando sugerindo que estas crianças estão concentradas em suas atividades; por outro lado, é uma das manifestações comportamentais do TPA(C) ${ }^{9}$.
Na Figura 2, é possível perceber a maior incidência de uso de chupeta em meninas, bem como a ocorrência de otites. Até o momento, não foram encontrados estudos que relacionem maior utilização de chupetas por meninas, mas a ocorrência de otites em usuários da chupeta, na presente amostra, corrobora outros estudos ${ }^{4}$ que relacionam tal hábito oral deletério com a predisposição para as otites. Dessa forma, a utilização da chupeta pode mudar a anatomia facial e causar uma mordida aberta anterior e, por consequência, a respiração oral. Além disso, frequentemente é possível observar que as crianças usuárias de chupeta e aleitamento artificial encontram-se dentre aquelas que fizeram pouco uso do aleitamento materno. Dessa forma, os respiradores orais podem apresentar maior susceptibilidade para as perdas auditivas condutivas mais evidenciadas nos quadros associados à hipertrofia de adenoamigdala do que nos de respiração oral funcional. As perdas auditivas condutivas em fase de aquisição da linguagem configuram um fator de risco para o TPA(C).

A Figura 3 mostra que as crianças mais velhas conseguem ler sozinhas, o que vem de encontro aos estudos que referem a relação da habilidade de leitura com a memória, a nomeação automática rápida e a consciência fonológica mais evidente em crianças mais velhas ${ }^{12,14}$. Da mesma forma, estas crianças conseguem recontar as histórias ordenadamente em relação às mais jovens que estão em níveis de educação anteriores, como mostrado também na Figura 4.

Comparando-se os níveis de educação, observou-se que as crianças do Jardim A foram as que mais tinham realizado exames audiológicos e as que menos assistiam à televisão. Podemos pensar se estes fatos não estão relacionados com o início da conscientização da importância de exames auditivos, conforme mencionado no início do capítulo, indicando que as crianças mais novas estão começando a ser beneficiadas pela identificação da necessidade da avaliação audiológica nos primeiros anos de vida evidenciadas pelos programas de triagem auditiva neonatal e pelos veículos de comunicação que tem divulgado o teste da orelhinha.

Por fim, a Figura 5 mostra as variáveis que podem constituir fatores de risco para o TPA(C) apontadas pelo resultado dos testes de correlação do presente estudo que são: necessidade de repetição de instruções, dificuldade de compreensão em ambientes competitivos e presença de irmãos.

A repetição de instruções e a dificuldade de compreensão em ambientes competitivos, são manifestações comportamentais do TPA(C) que podem ser relacionadas à atenção e memória ${ }^{9}$. 
Em relação à presença de irmãos, o presente estudo identificou a ocorrência de um número maior de fatores de risco para o TPA(C) nas crianças que possuem irmãos (figura 5). Apesar do estranhamento causado pelo referido resultado encontrou-se um estudo em que a prontidão escolar foi avaliada através dos seguintes domínios: identificação de cores e formas e formas copiadas, descrição de figuras, posição e reconhecimento espacial, identificação de números e contagem e identificação de letras e escrita. Como resultado, a variável quantidade de filhos correlacionou-se negativamente com os domínios identificação de números e contagem, identificação de cores e escrita e com o total de prontidão escolar. As autoras discutem que a prontidão escolar das crianças tende a ser maior em famílias com menor número de filhos ${ }^{24}$. Dessa forma, as variáveis que se correlacionaram negativamente com a quantidade de filhos no estudo pesquisado podem se encontrar relacionadas às variáveis atraso na aquisição de linguagem e dificuldades para memorizar nomes, lugares ou itinerários Tais variáveis foram elencadas, no presente estudo como fatores de risco para o TPA(C) ${ }^{9}$ além de envolver uma variável em comum relacionada à dificuldade com a nomeação das cores.

Há de se pensar que são necessárias campanhas de prevenção constantes para que se torne parte da cultura à realização de testes audiológicos. Esclarecimentos para outros profissionais da saúde e da educação sobre os sinais de alterações auditivas também se fazem necessárias como forma de prevenção de dificuldades escolares.

\section{CONCLUSÃO}

Evidenciou-se na pesquisa que a ocorrência do uso de chupeta foi alta nos participantes da pesquisa e que o aleitamento materno apesar de presente em quase todas as crianças ainda poderia ser prolongado. Além disso, a maioria das crianças não realizou nenhum tipo de exame audiológico e parte dessas solicita repetição de instruções com frequência.

Nas correlações, identificaram-se a utilização da chupeta e a maior freqüência de otites em meninas. Além disso, as crianças mais velhas realizam a leitura espontânea e recontam histórias ordenadamente em maior quantidade que as mais novas.

Identificaram-se fatores de risco estatisticamente significantes para o TPA $(C)$ nas variáveis: maior número de irmãos, maior solicitação de repetição de instruções e dificuldade de compreensão em ambiente competitivo para esta população.

\begin{abstract}
Purpose: to identify the risk factors for (Central) Auditory Processing Disorder in a group of 79 preschool children. Method: we applied a questionnaire to 79 parents or guardians of children attending preschool or first year of elementary school of the municipal, state and private schools in Porto Alegre. The questionnaire contained questions related to child development. Results: the research shows as the main results the use of a pacifier use $(73.4 \%)$; the duration of breastfeeding (36\%); the conduction of audiological testing (17.8\%); the time watching TV (59\%); and the number of repetitions of instructions (54\%); For the correlation tests the study showed the pacifier use $(p=0.006)$ and the higher incidence of otitis $(p=0.007)$ by girls. Older children performed spontaneous reading $(p=0.007)$ and retold stories neatly $(p=0.035)$. Conclusion: the risk factors for (Central) Auditory Processing Disorder were identified, being the variables: increased number of siblings $(p=0,005)$, more requesting for repeats $(p<0,001)$ and understanding difficulty in competitive environments $(p=0,002)$ as significant.
\end{abstract}

KEYWORDS: Hearing Loss, Central; Risk Factors; Child Development; Cognition; Child, Preschool

\section{REFERÊNCIAS}

1. ASHA: American Speech-Language-Hearing Association. (2005). (Central) Auditory Processing Disorders [Technical Report]. Available from www. asha.org/policy. [Acesso em: 11 de junho de 2009].
2. Gatto $\mathrm{Cl}$, Tochetto TM. Deficiência auditiva infantil: implicações e soluções. Rev CEFAC. Jan-mar, 2007; 9(1):110-5.

3. Balen AS, Bretzke L, Mottecy CM, Liebel G, Boeno MRM, Gondim LMA. Resolução temporal de 
crianças: comparação entre audição normal, perda auditiva condutiva e distúrbio do Processamento Auditivo. Rev Bras de Otorrinolaringol. 2009; 75 (1): 123-9.

4. Carrascoza KC, Possobon RF, Tomita LM, Moraes ABA. Consequences of bottle-feeding to the oral facial developement of initially breastfed children. J de Pediatr. 2006; 82 (5):395-7.

5. Ziliotto KN, Santos MFC, Monteiro VG, Hallinam MP, Moreira GA, Pereira LD et al. Avaliação do Processamento Auditivo em crianças com síndrome da apnéia/hipoapnéia obstrutiva do sono. Rev Bras de Otorrinolaringol. 2006; 72 (3): 321-7.

6. Santos JN, Lemos SMA, Rates SPM, Lamounier JA. Habilidades auditivas e desenvolvimento de linguagem em crianças. Pró-fono. out-dez, 2008; 20 (4): 255-60.

7. Santana CJ, Scopinho PAB, Ferreira RS, Simões TC, Santos JN. Conhecimento auditivo da população usuária do sistema único de saúde. Rev Soc Bras de Fonoaudiol. 2009; 14 (1): 75-82.

8. Joint Committee on Infant Hearing- YAER 2000 Position Statement: Principles and for early hearing detection and intervention programs. Am J Audiol.2000, 9 (1): 152-6.

9. Pereira LD. Identificação de desordem do processamento auditivo central através da observação comportamental: organização de procedimentos padronizados. In: SCHOCHAT, Eliane (Org.). Processamento Auditivo. São Paulo: Lovise; 1996. v.2 43-56.

10. Muniz LF, Roazzi A, Schochat E, Teixeira CF, Lucena JA. Avaliação da habilidade de resolução temporal, com uso de tom puro, em crianças com e sem desvio fonológico. Rev CEFAC. Out-dez, 2007; 9(4):447-62.

11. Tinius TP. The intermediate visual and auditory contínuos performance tests as a neuropsychological measure. Arch Clin Neuropsychol, 2003, 18 (2): 199-214.

12. Salgado C, Capellini SA. Desempenho em leitura e escrita de escolares com transtorno fonológico. Psicologia Escolar e Educacional. 2008; 8(2):179-88.

http://dx.doi.org/10.1590/S1516-18462011005000004

RECEBIDO EM: 27/04/2010

ACEITO EM: 17/08/2010

Endereço para correspondência:

Daniela Musskopf da Luz

Av: Francisco Talaia de Moura, 295

Porto Alegre - RS

CEP: 91170-090

E-mail: danimluz@ibest.com.br
13. Dadalto EV, Goldfeld M. Características comum à narrativa oral de crianças na pré-alfabetização. Revista CEFAC. Jan-mar , 2009; 11 (1), 42-9.

14. Capellini SA, Ferreira TL, Salgado CA, Ciasca SM. Desempenho de escolares bons leitores, com dislexia e com transtorno de déficit de atenção e hiperatividade em nomeação rápida. Rev Soc Bras de Fonoaudiol. 2007; 12 (2): 114-9.

15. Azevedo MF, Pereira, LD, Vilanova LCP, Goulart AL. Avaliação do processamento auditivo central: identificação de crianças de risco para alteração de linguagem e aprendizado durante o primeiro ano de vida. In: Marchesan IQ. et al. Tópicos em fonoaudiologia. São Paulo: Lovise; 1995. v.2, p.449.

16. Azevedo MF. Programa de prevenção e identificação precoce dos distúrbios da audição. In: Schochat E.(Org). Processamento Auditivo. São Paulo: Lovise, 1996. v.2 75-100.

17. Amorin MMR, Melo ASO. Avaliação dos exames de rotina no pré-natal. Rev Bras Ginec Obst. 2009; 31 (7): 367-74.

18. Victora CG. Intervenções para reduzir a mortalidade infantil pré-escolar e materna no Brasil. Rev Bras Epidemiol. 2001; 4: 369.

19. Marques MCS, Melo AM. Amamentação no alojamento conjunto. Rev CEFAC. Abr-jun, 2008; 10(2): 261-71.

20. Vasconcelos MGL, Lira PIC, Lima MC. Duração e fatores associados ao aleitamento materno em crianças menores de 24 meses de idade no estado de Pernambuco. Rev Bras Saúde Mater Infantil. 2006; 6: 99-105.

21. Araújo CMT, Silva GAP, Coutinho SB. A utilização de chupeta e o desenvolvimento sensório motor oral. Rev CEFAC. Abr-jun, 2009; 11(2): 261-7.

22. Petry C, Pereira UM, Pitrez PMC, Jones MH, Stein RT. Prevalência de sintomas de distúrbios respiratórios do sono em escolares brasileiros. J Pediatria. 2008; 84(2) 123-9.

23. Piovesan AJ, Yonamine RS, Lopes AS, Filho RC. Adiposidade corpórea e tempos de assistência à TV em escolares de 11 a 14 anos de duas regiões geográficas do município de Campo Grande- MS. Rev. Bras de Cineantropometria \& Desempenho Humano. 2002; 4(1):17-24.

24. Andrada EGC, Bárbara SR, Gabriela BC, Benetti IC. Fatores de risco e proteção para a prontidão escolar. Psicologia, Ciência e Profissão. 2008; 28 (3): 536-7.

25. Sandri MA, Meneghetti SL, Gomes E. Perfil comunicativo de crianças entre 1 e 3 anos com desenvolvimento normal de linguagem. Rev CEFAC. Jan-mar, 2009; 11 (1): 34-41. 